\title{
Interacting Intersecting Masers
}

\author{
Shuji Deguchi \\ Nobeyama Radio Observatroy, National Astronomical Observatory, \\ Minamisaku, Nagano 384-13, Japan
}

\begin{abstract}
An interaction of two masers through plasma longitudinal waves in the Stimulate Raman Scattering has been investigated and applied for the extreme high-velocity $\left(900 \mathrm{~km} \mathrm{~s}^{-1}\right)$ water masers discovered in NGC 4258. For the stimulated Raman scattering, the frequency down-shifted components are easily created but not the up-shifted components. We use the interacting masers for the explanation for the presence of the up-shifted components. The interaction occurs when the wave vector of the longitudinal plasma wave associated with one incident maser coincides with the wave vector of the other longitudinal wave associated with the other maser. This interaction produces two daughter waves, frequency up-shifted and down-shifted radiations with a shift of roughly a plasma frequency. This interaction occurs when two masers intersect with intersecting angle of about 176 degree in plasma of the electron density of about $510^{6} \mathrm{~cm}^{-3}$. The brightness temperature of the original incident masers must be greater than about $10^{13} \mathrm{~K}$ (depending on the line width, and the maser beaming angle, etc.) and the scale length of plasma must be larger than about $10^{13} \mathrm{~cm}$.
\end{abstract}

\section{Extreme high-velocity $\mathrm{H}_{2} \mathrm{O}$ masers}

Very powerful water masers from nuclei of galaxies have been known for 10 years (Claussen \& Lo 1986). Recently, Nakai et al. (1992) have found that the water masers from the galaxy NGC4258 contain weak emission at extreme high velocity $\left(\Delta V=900 \mathrm{~km} \mathrm{~s}^{-1}\right)$. The spectra of the extreme high-velocity features are composed of narrow spikes of the width of about $10 \mathrm{~km} \mathrm{~s}^{-1}$, indicating that these are maser emission. The intensities of the lower frequency features (higher velocity components) are about $1 / 3$ to $1 / 10$ and the intensities of the higher frequency fealures (lower velocity components) about $1 / 10$ to $1 / 30$. No noticable emission is found at the middle betweeen main and these features in the velocity width of about $700 \mathrm{~km} \mathrm{~s}^{-1}$.

The high velociy shift of water masers in NGC 4258 faces up to a difficulty when we explain it by the Doppler motion of the gas. The gas outflow of the order of $1000 \mathrm{~km} \mathrm{~s}^{-1}$ is compared to the supemova ejecta. It produces a strong shock in the ambient medium and would destroy all molecules. An altemative possibility is an acceretion disk with the rotating velocity of about $1000 \mathrm{~km} \mathrm{~s}^{-1}$. However, the kinetic temperature of water maser cloud must be below $2000 \mathrm{~K}$. It would be difficult to sustain such unusually fast rotating disk without ionizing the gas in the disk.

\section{Stimulated Raman scattering}

In this paper we pursue a possibility of non-Doppler explanations of the extreme high-velocity features, i. e., the stimulated Raman scattering in plasma. The Raman scattering produces photons with up- and down-shifted frequencies separated roughly by the plasma frequency (Radhakrishnan et al. 1975). The model requires the compact HIl regions of the density Ne=107 $\mathrm{cm}^{-3}$ and the scale length $r=10^{13} \mathrm{~cm}$. The Raman scattering in the plasma has been investigated by Fernandez \& Reinisch (1978), and Burdjuzha et al. (1979). However, their one dimensional model (forward scattering) cannot successfully account the intensity of the higher velocity features. 
We have reinvestigated the stimulated Raman process based on the dispersion relation analysis and show that the Raman scattering occurs at a reasonable intensity level of observed masers. Here, we simply summarize the results.

The dispersion relations of the plasma longitudinal and transverse waves and the resonance conditions lead the velocity shift in the case of back scattering as

$\left(\Delta \mathrm{V} / 900 \mathrm{~km} \mathrm{~s}^{-1}\right)^{2}=0.11\left(\mathrm{Ne}_{\mathrm{e}} / 5.910^{6} \mathrm{~cm}^{-3}\right)+0.89\left(\mathrm{~T}_{\mathrm{e}} / 4000 \mathrm{~K}\right) \cos ^{2} \theta_{3}$

where $\theta_{3}$ is the angle of scattering for the plasma wave. Because the temperature of the plasma cannot be decreased well below $4000 \mathrm{~K}$, this equation gives the lower limit for the line separation. So that the observed fact, i.e., the absence of emission between extrem-high and main features, can well be understood by this theory.

The propagation equations of electromagnetic waves (Maxwell's equations) and the local anlysis lead the Raman instability condition for the back scattering as

$$
\begin{gathered}
\left(\mathrm{T}_{\mathrm{b}} / 5.610^{11} \mathrm{~K}\right)\left(\Delta \Omega_{0} / 10^{-4}\right)\left(\Delta \mathrm{v}_{0} / 1 \mathrm{~km} \mathrm{~s}^{-1}\right)\left(5.910^{6} \mathrm{~cm}^{-3} / \mathrm{N}_{\mathrm{e}}\right)^{3} \\
\times\left(\mathrm{T}_{\mathrm{e}} / 4000 \mathrm{~K}\right)^{3}\left(\Delta \mathrm{V} / 900 \mathrm{~km} \mathrm{~s}^{-1}\right) \cos ^{2} \psi_{1} \cos ^{2} \theta_{3}>1
\end{gathered}
$$

This inequality assures that the Raman backward scattering occurs easily in the astrophysical plasma.

\section{Necessity for the interacting masers}

Up-shifted photons cannot be created by backward scattering. This is because the up-shifted photons must consume the plasma longitudinal waves and this is possible only by the forward scattering. The efficiency of the forward scattering is about several orders of magnitude lower than that of the backward scattering because of the consumption of plasma waves. It requires $\mathrm{Tb}$ of about $10^{21} \mathrm{~K}$ and it is impossible to obtain in the astrophysical masers.

To create the up-shifted photons, we introduce the other maser interacting with the original maser through plasma waves. We consider another maser incident from the other direction in such a way that it produces the same plasma wave created by original masers. This is possible at the beam crossing angle of $176 \mathrm{deg}$. The formulation can be made in a parallel way as the case of one incident maser. The final unstable condition is obtained and it is very similar with that of the former equation (2) given above. This process occurs when the original maser is stronger than the second incident maser. These are the intersecting interacting masers through plasma longitudinal waves. Interacting masers have been considered before by Deguchi \& Watson (1989) to explain the extreme brightness of W49 masers.

\section{Further developement}

Above theory tells us that the Raman maser gain depends on the angle of linear polarization. Small linear polarization is expected from this theory (of about $10 \%$ for the scattering angle of 5 or 175 degree in the unsaturated maser). It is known that the center components of the extremely powerful $\mathrm{H}_{2} \mathrm{O}$ masers are not polarized and the measurements of linear polarization for the up- and down-shifted components would prove the validity of the Raman maser model.

\section{References}

Burdjuzha, V. V., Charugin, V. M., \& Tomozov, V. M., 1979, AAp, 79, 306

Claussen, M. J., \& Lo, K. Y. 1986, ApJ, 308, 592

Deguchi, S., \& Watson, W. D. 1989, ApJ, 340, L17

Fernandez, C., \& Reinisch, G. 1978, AAp, 67, 163

Nakai, N., Inoue, M., \& Miyoshi, M., 1992, (preprint)

Radhakrishnan, V., Goss, W. M., \& Bhandari, R. 1975, Pramana, 5, 49 